\title{
Kinesioterapia respiratoria en la bronquiolitis aguda: estrategia terapéutica, bases fisiológicas e impacto clínico
}

\author{
Klgo. Iván Rodríguez',2, Klgo. Claudio Báez², \\ Klga. Tania Contreras ${ }^{2}$, Dr. Daniel Zenteno',3 \\ I Unidad de Ventilación Mecánica Prolongada-Programa de Rehabilitación Respiratoria Infantil. Servicio de Pediatría. \\ Hospital Dr. Guillermo Grant Benavente. Concepción. \\ 2 Grupo de Investigación en Kinesiología Cardiorrespiratoria. Escuela de Kinesiología, Facultad de Ciencias de la Salud. \\ Universidad San Sebastián. Concepción. \\ 3 Departamento de Pediatría, Facultad de Medicina. Universidad de Concepción.
}

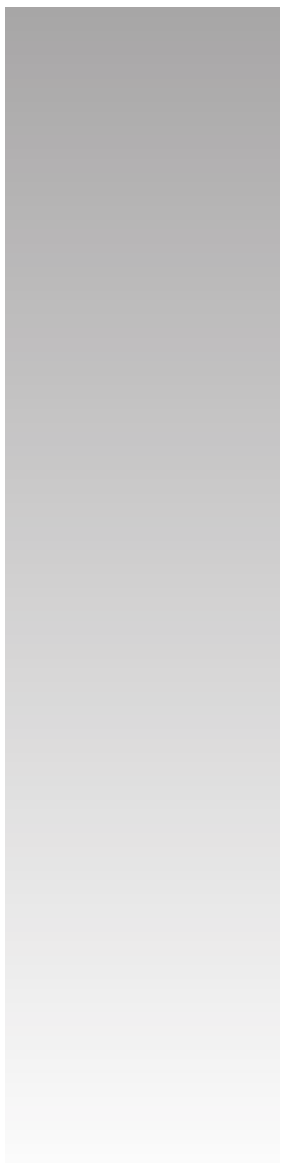

\section{CHEST PHYSICAL THERAPY IN ACUTE BRONCHIOLITIS: THERAPEUTIC STRATEGIES, PHYSIOLOGICAL BASIS AND CLINICAL IMPACT}

Acute bronchiolitis $(A B)$ is a common acute respiratory disease that affects children younger than 2 years. The main etiological factor is given by respiratory syncytial virus. The role of chest physical therapy (CPT) in the management of this condition has been studied in recent years with discrepant results because conventional CPT has been designed for use in adult patients, which significantly compromises external validity of the studies that have evaluated the effectiveness of these treatment protocols in infants. In this sense prolonged slow expiration (PSE) arises as a therapeutic alternative, since the physiological purpose of its implementation responds to the mechanism by which the respiratory function deteriorates in this patient's group. In recent years, successive reports have been published regarding the benefits PSE technique on the clinical course of patients with $A B$, which have shown that helps to reduce the hyperinflation and improve airway clearance in infant, without triggering dynamic hyperinflation. In the present review were addressed in an integrated manner the most relevant aspects related to bronchial obstruction mechanism in infant, physiological effect and impact of PSE. This report intended to be a contribution to advance the development of future research that will contribute to enrich the knowledge of this discipline.

Key words: Acute bronchiolitis, chest physical therapy, lung function, infant.

\section{RESUMEN}

La bronquiolitis aguda (BA) es una enfermedad respiratoria aguda frecuente que afecta a niños menores de 2 años, siendo su principal agente etiológico el virus respiratorio sincicial. El rol de la kinesioterapia respiratoria (KTR) en el manejo de esta patología ha sido materia de estudio en los últimos años con resultados discrepantes debido a que las técnicas de KTR convencional han sido diseñadas para su aplicación en el paciente adulto, lo que compromete significativamente la validez externa de los estudios que han evaluado la efectividad de estos protocolos terapéuticos en lactantes. En este contexto, surge la técnica de espiración lenta prolongada (ELPr) como alternativa terapéutica, puesto que el sustrato fisiológico de su ejecución responde al mecanismo mediante el cual se deteriora funcionalmente la mecánica ventilatoria en este grupo de pacientes. En los últimos años, se han publicado reportes consecutivos respecto a los beneficios de la técnica de ELPr sobre el curso clínico de los pacientes con BA, donde se ha observado contribuye a reducir la hiperinsuflación y permeabilizar la vía aérea del lactante, sin gatillar hiperinsuflación dinámica; pilares claves en la disfunción respiratoria en estos pacientes. En la presente revisión se abordaron de manera integrada los aspectos más relevantes vinculados a mecanismo de obstrucción bronquial del lactante, efecto fisiológico de la ELPr e impacto cínico de su ejecución. Datos que pretenden ser un aporte para avanzar en el desarrollo de investigaciones futuras que contribuyan a enriquecer el conocimiento de esta disciplina.

Palabras clave: Bronquiolitis obliterante, terapia respiratoria, función pulmonar, lactantes.

Correspondencia: Klgo. Iván Rodríguez Núñez MSc. Servicio de Pediatría,

Hospital Dr. Guillermo Grant Benavente.

San Martín 1436 Concepción, Región del Bío-Bío, Chile.

Teléfonos: 04 | 2687489 - 04| 2722630.

E-mail: klgo.ivanronu@gmail.com

Conflicto de interés: Los autores de la revisión declaran no poseer conflicto de interés. 


\section{INTRODUCCIÓN}

La bronquiolitis aguda (BA) es una enfermedad respiratoria aguda frecuente que afecta a niños menores de 2 años generado por inflamación de las vías respiratorias bajas que da lugar a un cuadro clínico de dificultad respiratoria, generalmente precedido de síntomas de afectación de vías altas como coriza y tos. El principal factor etiológico está dado por la infección viral, siendo el virus respiratorio sincicial (VRS), adenovirus, influenza A y B y parainfluenza I, 2 y 3; los agentes más importantes ${ }^{(1)}$.

En nuestro país, los cuadros bronquiales agudos constituyen la principal causa específica de morbilidad pediátrica, incidencia que muestra una gran variabilidad estacional relacionada con factores de riesgo, como son las infecciones virales, el frío y la contaminación atmosférica ${ }^{(2)}$. El diagnóstico de BA se establece basado en la historia y examen físico del paciente, caracterizado por la presentación de síntomas prodrómicos en vía aérea superior, seguidos por sibilancias y signos de aumento de trabajo respiratorio. En pacientes lactantes, el curso clínico de la BA es dinámico y oscila desde eventos transitorios tal como apnea o hipersecreción nasal; a fallo respiratorio progresivo debido a la obstrucción de la vía aérea pequeña ${ }^{(3)}$. Adicionalmente, la severidad de la enfermedad puede ser valorada a través de diversos sistemas de puntuación. En este ámbito, el escore modificado de Tal es uno de los más ampliamente utilizado en nuestro país debido a su buena confiabilidad, validez y correlación con saturación de oxígeno en niños menores de 2 años $(2,4,5)$.

El rol de la KTR en el manejo de esta patología ha sido materia de estudio en los últimos años con resultados discrepantes. En este contexto, Bohe y colsen, un ensayo clínico con asignación aleatoria (ECA), evaluó el efecto de la KTR en 32 niños menores a dos años con diagnóstico de $\mathrm{BA}$, donde no observó mejoría significativa en el score clínico ni tampoco reducción de los días de hospitalización en el grupo de tratamiento(6); más tarde Gajdos y cols en un ECA controlado y multicéntrico donde evaluó la eficacia de la KTR en 496 lactantes hospitalizados por BA, no observó reducción significativa en los días de hospitalización, utilización de oxígeno, ni incidencia de complicaciones; incluso observó un mayor riesgo de vómitos y desestabilización respiratoria transitoria en el grupo experimental (Riesgo relativo $=10)^{(7)}$. Recientemente Roqué y cols, realizaron una revisión sistemática y meta análisis de ensayos clínicos donde concluyeron que la KTR no atenúa la severidad de los signos respiratorios, no reduce la estancia hospitalaria, ni disminuye los requerimientos de oxígeno en lactantes hospitalizados con $\mathrm{BA}^{(8)}$.

En la mayoría de los ECA, las técnicas kinésicas evaluadas corresponden a lo que se denomina "KTR convencional", compuesta por: drenaje bronquial, vibración, percusión, espiración forzada, succión nasotraqueal, empleo de presión positiva espiratoria (PEP), tos asistida, entre otras $(9,10)$. Todas estas técnicas han sido diseñadas para su aplicación en el paciente adulto, lo que compromete significativamente la validez externa de los estudios que han evaluado la efectividad de estos protocolos terapéuticos en lactantes.

En términos fisiopatológicos, la BA se caracteriza por inflamación aguda, edema, hipersecreción de mucus y bronco- espasmo, lo que afecta al flujo y permeabilidad de la vía aérea pequeña generando sibilancias, hiperinsuflación y atelectasias en los lactantes que padecen $B A^{(3)}$. En virtud de lo anterior, las técnicas de KTR deben ser enfocadas en corregir el deterioro funcional del sistema respiratorio causado por este cuadro clínico. En este contexto, surge la técnica de espiración lenta prolongada (ELPr) como alternativa terapéutica, puesto que el sustrato fisiológico de su ejecución responde al mecanismo mediante el cual se deteriora funcionalmente la mecánica ventilatoria en este grupo de pacientes ${ }^{(1)}$.

En los últimos años se han publicado reportes consecutivos respecto a los beneficios de la técnica de ELPr sobre el curso clínico de los pacientes con $B A^{(11-16)}$. Sin embargo, las bases fisiológicas y el impacto clínico de la ejecución de esta técnica kinésica, han sido escasamente abordados de manera integral. Razón por la cual los objetivos de esta revisión son describir el deterioro funcional causado por la obstrucción bronquial, las bases fisiológicas de la ejecución de la ELPr y discutir el impacto de la ejecución de esta técnica sobre el curso clínico de la BA.

\section{MECANISMO DE OBSTRUCCIÓN E IMPLICANCIA FISIOLÓGICA}

Desde el punto de vista físico nuestro aparato respiratorio constituye un sistema dinámico que modifica de manera continua la geometría del parénquima pulmonar y vía aérea de conducción a lo largo del ciclo respiratorio. Para realizar esta función, es necesario que el sistema muscular sea capaz de vencer la carga impuesta por la resistencia de la vía aérea y la elastancia toracopulmonar, con el propósito de generar un flujo tal que permita un movimiento de volumen de entrada y salida de aire, para así asegurar una ventilación alveolar que permita un intercambio adecuado de gases ${ }^{(17)}$.

En el lactante, las zonas de mayor resistencia de la vía aérea extratorácica, están constituidas por la vía naso y orofaríngea que corresponde al $40 \%$ de la resistencia total de las vías respiratorias; y en la vía aérea intratorácica, el lugar principal de resistencia al flujo está constituido por las pequeñas vías respiratorias en una proporción que llega al 50\% de las resistencias totales, mientras que en el adulto no supone más que $10 \%$. Por este motivo, las pequeñas vías respiratorias en los niños pequeños se ven más afectadas por un estrechamiento funcional que las de un niño mayor o un adulto, desarrollando de esta forma rápidamente cuadros clínicos y funcionales de obstrucción graves ${ }^{(18,19)}$.

En la BA, una vez que se ha instaurado la infección, en etapas precoces se produce necrosis del epitelio respiratorio seguida de proliferación del epitelio bronquiolar y destrucción de las células ciliadas (principalmente por VRS). Posteriormente se genera la infiltración peribronquiolar de linfocitos, macrófagos y células plasmáticas, así como también, formación de edema de la submucosa y adventicia. Esto conlleva a alteración severa en el transporte mucociliar, hipersecreción y aumento en la resistencia de la vía aérea bronquiolar. La obstrucción de las vías aéreas pequeñas da lugar a un patrón de distribución irregular de áreas insufladas y atelectásicas lo que da origen a la alteración en la relación ventilación- 
perfusión, disminución crítica del diámetro bronquiolar en espiración y atrapamiento aéreo; provocando un aumento de los volúmenes pulmonares (volumen residual, capacidad residual funcional y capacidad pulmonar total) y disminución de la compliance pulmonar lo que, asociado al aumento de resistencia de la vía aérea, genera un aumento significativo de las constantes de tiempo del sistema respiratorio, constituyendo el sustrato fisiopatológico del aumento del trabajo muscular tanto inspiratorio como espiratorio en este grupo de pacientes ${ }^{(1,3)}$.

\section{ESTRATEGIA TERAPÉUTICA: ESPIRACIÓN LENTA PROLONGADA}

\section{Bases fisiológicas}

La técnica de ELPr fue desarrollada específicamente para niños menores a dos años, tomando en consideración sus características fisiológicas y mecanismo de obstrucción en la BA. En esta técnica, el kinesiólogo posiciona la región hipotenar de una mano sobre el tórax del niño (bajo la horquilla esternal) y la región hipotenar de la otra mano en el abdomen (bajo el ombligo); luego, visualmente identifica las fases inspiratorias y espiratorias, y al final de esta última se ejerce una presión manual en el tórax y abdomen sin producir aceleración de flujo espiratorio. La compresión desde el tórax se ejerce en dirección craneal-caudal y la presión desde el abdomen se ejerce en dirección caudal-craneal. Esto prolonga la fase espiratoria lo que permite, por una parte, promover la salida de las secreciones bronquiales desde el sistema respiratorio del niño, y por otro aumentar el volumen corriente espirado con el propósito de contrarrestar la hiperinsuflación raíz de la obstrucción bronquiolar, sin generar el fenómeno de compresión dinámica en la vía aérea ${ }^{(15,16)}$.

Se ha demostrado, durante la ejecución de la ELPr, que debido a la restricción externa impuesta por la compresión manual sobre el sistema toracopulmonar, el volumen corriente inspirado (VTi) disminuye un 40\%, lo que contribuye a prolongar el tiempo espiratorio. Por otra parte, se han observado tasas de disminución del volumen de reserva espiratoria (\% VRE) que oscilan entre 32\% y 53\%, durante la ELPr, así como también, aumentos del volumen corriente exhalado (VTe) del 25\% posterior a cada secuencia de ELPr, todo esto sin cambios significativos de la compliance toracopulmonar. En adición a esto, el porcentaje de vaciamiento pulmonar es inversamente proporcional a la edad, en lactantes, a medida que aumenta su edad, menos efectiva es la ELPr para aumentar el volumen exhalado(12,15). Esta reducción del volumen pulmonar es fisiológicamente asociada con los reflejos protectores del sistema respiratorio, los cuales restauran los volúmenes pulmonares a través de suspiros, fenómeno denominado reflejo de Hering-Breuer. Durante la ELPr el lactante exhala una cantidad suficiente de aire para gatillar este reflejo, el que es más fácilmente inducido en lactantes más pequeños debido a la inmadurez de los receptores del sistema respiratorio; mientras que aquellos con enfermedades respiratorias crónicas son menos proclives a generarlo debido a la alteración pulmonar(12,20) (Figura I).

\section{Impacto clínico}

Se ha demostrado que, a través de la técnica de ELPr, es posible reducir el valor de la frecuencia respiratoria, frecuencia cardíaca e intensidad de las retracciones posterior a la intervención; lo que permite reducir de manera significativa el score clínico de severidad del cuadro. Adicionalmente, se ha observado que aquellos niños tratados con ELPr, una mejoría precoz de los signos y síntomas, sin que eso constituyera una reducción en los días de hospitalización"'.

Recientemente Gomes y cols, en un ECA, evaluó la efectividad de la ELPr en 30 lactantes con diagnóstico de

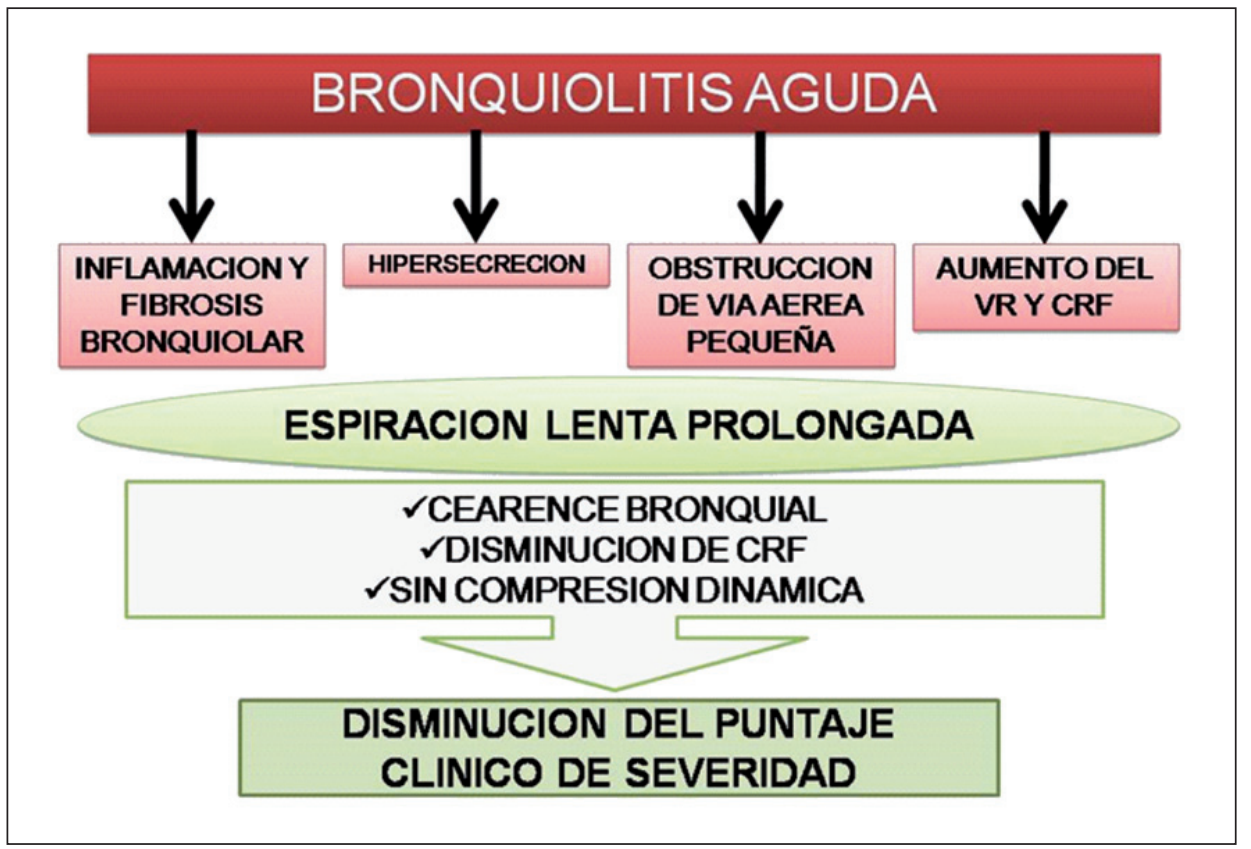

Figura I. Esquema del efecto de la técnica de ELPr sobre los componentes fisiopatológicos de la BA. 


\section{Tabla I. Resumen de la evidencia sobre el efecto de la KTR en la BA}

\begin{tabular}{|c|c|c|}
\hline Autor & Diseño/Intervención & Resultado \\
\hline $\begin{array}{l}\text { Bohe L, et al. } \\
(2004)^{6}\end{array}$ & $\begin{array}{l}\text { ECA/ En grupo experimental }(n=16) \text { se realizó drenaje, } \\
\text { percusiones, vibraciones, succión nasofaríngea; grupo } \\
\text { control }(n=16) \text { se realizó sólo succión nasofaríngea }\end{array}$ & $\begin{array}{l}\text { - No se observaron diferencias en el escore clínico ni en } \\
\text { los días de hospitalización }\end{array}$ \\
\hline $\begin{array}{l}\text { Gajdos V, et al. } \\
(2010)^{7}\end{array}$ & $\begin{array}{l}\text { ECA/ En grupo experimental ( } n=246 \text { se realizó acelera- } \\
\text { ción de flujo espiratorio, estimulación de la tos y succión } \\
\text { nasotraqueal; grupo control ( } n=250 \text { se realizó sólo } \\
\text { succión nasotraqueal }\end{array}$ & $\begin{array}{l}\text { - No se observaron diferencias en los días de hospitaliza- } \\
\text { ción } \\
\text { - Frecuencia de vómitos y desestabilización respiratoria } \\
\text { fue mayor en grupo experimental }\end{array}$ \\
\hline $\begin{array}{l}\text { Postiaux } G \text {, et al. } \\
(2011)^{\prime \prime}\end{array}$ & $\begin{array}{l}\text { ECA/En grupo experimental }(n=12) \text { se realizó ELPr; } \\
\text { grupo control }(n=8) \text { se realizó nebulización con suero } \\
\text { hipertónico }\end{array}$ & $\begin{array}{l}\text { - En el grupo ELPr se observó menor, escore clínico, } \\
\text { sibilancias, frecuencia cardíaca } \\
\text { - Sin eventos adversos }\end{array}$ \\
\hline $\begin{array}{l}\text { Lanza F, et al. } \\
(2011)^{12}\end{array}$ & Cuasi experimental/ ELPr en lactantes sibilantes, $\mathrm{n}=18$ & $\begin{array}{l}\text { - Disminución del Vti, sin cambios en el PEF } \\
\text { - Aumento del volumen exhalado }\end{array}$ \\
\hline $\begin{array}{l}\text { Sánchez } M \text {, et al. } \\
(20 \mid 2)^{13}\end{array}$ & $\begin{array}{l}\text { ECA/ Grupo experimental }(n=138) \text { se realizó ELPr, } \\
\text { vibraciones manuales y tos provocada; grupo control } \\
(n=144) \text { se realizó maniobras placebo }\end{array}$ & $\begin{array}{l}\text { - No hubo disminución de la estancia hospitalaria ni de } \\
\text { uso de } \mathrm{O}_{2} \text { en muestra total } \\
\text { - Aquellos con estudio positivo para VRS necesitaron } \\
\text { menos horas de } \mathrm{O}_{2}\end{array}$ \\
\hline $\begin{array}{l}\text { Gomes É, et al } \\
(2012)^{14}\end{array}$ & $\begin{array}{l}\text { ECA/ La muestra fue distribuida en } 3 \text { grupos; grupo KTR } \\
\text { convencional }(n=10) \text {, grupo } \operatorname{ELP}(n=10) \text { y grupo } \\
\text { succión de vía aérea superior }(n=10)\end{array}$ & $\begin{array}{l}\text { - El escore clínico disminuyó en grupo KTR convencional } \\
\text { y ELPr posterior a la intervención } \\
\text { - } 72 \text { h posteriores a la intervención se observó mejoras } \\
\text { sólo en el grupo ELPr }\end{array}$ \\
\hline $\begin{array}{l}\text { Rochat I, et al. } \\
(20 \mid 2)^{2 !}\end{array}$ & $\begin{array}{l}\text { ECA/ Grupo experimental }(n=50) \text { se realizó ELPr, acele- } \\
\text { ración de flujo espiratorio y tos provocada; grupo control } \\
(n=49) \text { succión rinofaríngea }\end{array}$ & $\begin{array}{l}\text { - No hubo diferencia en el tiempo de recuperación de la } \\
\text { - Estabilidad clínica } \\
\text { - Escore clínico de gravedad mostró una mejora más rápi- } \\
\text { da cuando se incorporaron propiedades estetoacústicas }\end{array}$ \\
\hline $\begin{array}{l}\text { De Córdoba F, et al. } \\
(2013)^{15}\end{array}$ & $\begin{array}{l}\text { Cuasi experimental/ I } 8 \text { Lactantes con sibilancias recurren- } \\
\text { tes sin patología respiratoria aguda sometidos a KTR con } \\
\text { ELPr }\end{array}$ & $\begin{array}{l}\text { - En la muestra se observa aumento de VTe, reducción de } \\
\text { FR, sin cambios en el PEF y sin complicaciones posterior } \\
\text { a la intervención }\end{array}$ \\
\hline
\end{tabular}

ECA: Ensayo clínico con asignación aleatoria; ELPr: Espiración lenta prolongada; VTi: Volumen corriente inhalado; VTe: Volumen corriente exhalado; PEF: Flujo espiratorio peak; KTR: Kinesioterapia respiratoria; VRS: Virus respiratorio sincicial.

BA por VRS, quienes fueron clasificados en 3 grupos; uno recibió KTR a través de $\operatorname{ELP} \operatorname{Pr}(\mathrm{n}=10)$, otro KTR convencional $(n=10)$ (vibraciones, compresión espiratoria, drenaje postural modificado, clapping) y el último sólo succión naso traqueal $(n=10)$. Los resultados de este estudio mostraron que aquellos pacientes tratados tanto con la técnica de ELPr como con KTR convencional tuvieron un menor score clínico después de la intervención, sin embargo, sólo aquellos tratados con ELPr mostraron mejoría significativa 72 h posterior a la hospitalización $n^{(14)}$. Por otra parte, Sánchez y cols, en un ECA y doble enmascaramiento realizado con 236 lactantes menores a 7 meses hospitalizados por diagnóstico de BA (primer episodio), donde evaluó la eficacia de la ELPr contra una intervención placebo (sólo drenaje postural), no observó diferencias significativas en la estancia hospitalaria, ni en los días de utilización de oxígeno en la muestra general. No obstante, en aquellos pacientes con estudio positivo para VRS, se observó una menor utilización de oxígeno durante la hospitalización (48,8 h en el grupo ELPr vs 58,68 h en el grupo placebo $p=0,042)^{(13)}$.

En términos generales, cuando las técnicas de kinesioterapia respiratoria han sido empleadas de manera integral, es cuando más significativos han sido las mejoras reportadas en la función pulmonar y curso clínico de la enfermedad. En cambio, en niños menores a dos años, cuando la técnica de ELPr se emplea en asociación a otros procedimientos (como la aceleración de flujo espiratorio) no muestra los resultados observados en puntaje de gravedad y pronóstico del cuadro clínico ${ }^{21}$. Lo que hace suponer la existencia de una estrecha asociación entre las bases físicas de la técnica kinésica, las características anátomo funcionales del lactante y la respuesta del paciente a la terapia; aspecto que no ha sido evaluado en su totalidad, a través de estudios experimentales (Tabla I).

\section{CONCLUSIÓN}

En los últimos años, la kinesiología respiratoria ha sufrido avances sustanciales gracias a los progresos tecnológicos en el área de la evaluación, terapia y rehabilitación; puesto que ha sido posible automatizar y estandarizar los procesos de medición de variables, lo que ha permitido desarrollo de investigación clínica en este campo con altos estándares de validez y confiabilidad. Esto nos ha aportado datos de gran relevancia para conocer el sustento físico y efectos fisiológicos de las estrategias terapéuticas para abordar las distintas entida- 
des nosológicas que afectan al sistema respiratorio. Por este motivo, el proceso de revisión sistemática, debe realizarse teniendo en cuenta no solamente el nivel de evidencia, sino que también la validez interna que tuvo proceso de investigación desarrollado, las características anátomo funcionales del paciente en estudio, el tipo de técnica empleada y validez externa de los resultados obtenidos. Con este enfoque será posible desarrollar conclusiones adecuadas y establecer recomendaciones basados en antecedentes empíricos actuales con un alto nivel de calidad metodológica, propósito central de la medicina basada en evidencia.

Dado que el niño, en términos anátomo funcionales, no se comporta como un adulto pequeño, las estratégicas terapéuticas deben ser adaptadas a los aspectos fisiopatológicas que sustentan la enfermedad respiratoria del paciente en cuestión. Razón por la cual, la ELPr ha demostrado ser una técnica efectiva para disminuir los signos y síntomas de los pacientes con BA, gracias a que se enfoca en reducir la hiperinsuflación y permeabilizar la vía aérea del lactante, sin gatillar hiperinsuflación dinámica; pilares claves en la disfunción respiratoria en estos pacientes.

En la presente revisión se abordaron de manera integrada los aspectos más relevantes vinculados a mecanismo de obstrucción bronquial del lactante, efecto fisiológico de la ELPr e impacto cínico de su ejecución. Datos que pretenden ser un aporte para la construcción de hipótesis y preguntas de investigación con el fin de avanzar en el desarrollo de investigaciones futuras que contribuyan a enriquecer el conocimiento de esta apasionante disciplina.

\section{AGRADECIMIENTOS}

A María Andrea Arredondo Avendaño (bibliotecóloga Universidad San Sebastián, campus Concepción) por el valioso apoyo bibliográfico prestado para el desarrollo de esta revisión.

\section{REFERENCIAS}

I. Crespo M, Crespo-Marcos D. Bronquiolitis. En: Cruz-Hernández M, Jiménez R, Editores, Nuevo Tratado de Pediatría. Barcelona, España: Editorial Océano Ltda.; 2009. p. I420-7.

2. Ministerio de Salud de Chile. Guía Clínica: Infección Respiratoria Aguda Baja de Manejo Ambulatorio en Menores de 5 Años. MINSAL 2013. Disponible en: www.minsal.cl [Consultado el 05 de agosto de 2013].

3. American Academy of Pediatrics. Subcommittee on Diagnosis and Management of Bronchiolitis. Diagnosis and Management of Bronchiolitis. Pediatrics 2006; I I 8: 1774-93.

4. McCallum GB, Morris PS, Wilson CC, Versteegh LA, Ward LM, Chatfield MD, et al. Severity scoring systems: are they internally valid, reliable and predictive of oxygen use in children with acute bronchiolitis? Pediatr Pulmonol 2013; 48: 797-803.
5. Pavón D, Castro-Rodríguez JA, Rubilar L, Girardi G. Relation between pulse oximetry and clinical score in children with acute wheezing less than 24 months of age. Pediatr Pulmonol 1999; 27: 423-7.

6. Bohé L, Ferrero ME, Cuestas E, Polliotto L, Genoff M. Indicación de fisioterapia respiratoria convencional en bronquiolitis aguda. Medicina (Buenos Aires) 2004; 64: 198-200.

7. Gajdos V, Katsahian S, Beydon N, Abadie V, de Pontual L, Larrar $S$, et al. Effectiveness of chest physiotherapy in infants hospitalized with acute bronchiolitis: a multicenter, randomized, controlled trial. PLoS Med 20 I 0; 7 (9): el 000345.

8. Roquéi Figuls M, Giné-Garriga M, Granados Rugeles C, Perrotta C. Chest physiotherapy for acute bronchiolitis in paediatric patients between 0 and 24 months old. Cochrane Database Syst Rev 20 I2; 2: CD004873.

9. Ides K, Vissers D, De Backer L, Leemans G, De Backer W. Airway clearance in COPD: need for a breath of fresh air? A systematic review. COPD 201।; 8: 196-205.

10. Pryor JA. Physiotherapy for airway clearance in adults. Eur Respir J 1999; 14: 1418-24

I I. Postiaux G, Louis J, Labasse HC, Gerroldt J, Kotik AC, Lemuhot $A$, et al. Evaluation of an alternative chest physiotherapy method in infants with respiratory syncytial virus bronchiolitis. Respir Care 2011; 56: 989-94.

12. Lanza F, Wandalsen G, Dela Bianca AC, Cruz CL, Postiaux G, Solé D. Prolonged slow expiration technique in infants: effects on tidal volume, peak expiratory flow, and expiratory reserve volume. Respir Care 201 I; 56: 1930-5.

13. Sánchez M, Martín Martín R, Cano Fernández J, Martínez Sánchez G, Gómez Martín J, Yep Chullen G, et al. Chest physiotherapy and bronchiolitis in the hospitalised infant. Double-blind clinical trial. An Pediatr (Barc) 2012; 77: 5-11.

14. Gomes EL, Postiaux G, Medeiros DR, Monteiro KK, Sampaio LM, Costa D. Chest physical therapy is effective in reducing the clinical score in bronchiolitis: randomized controlled trial. Rev Bras Fisioter 2012; 16: 241-7.

15. De Cordoba F, Wandalsen GF, da Cruz CL, Solé D. Impact of the prolonged slow expiratory maneuver on respiratory mechanics in wheezing infants. J Bras Pneumol 20 13; 39: 69-75.

16. Postiaux G, Zwaenepoel B, Louis J. Chest physical therapy in acute viral bronchiolitis: an updated review. Respir Care 20 I3; 58: | 54 I5.

17. West J. Fisiología respiratoria. 7 ed. Editorial Médica Panamericana; 2007.

18. Postiaux G. Fisioterapia respiratoria en el niño. McGraw-Hill; 2000.

19. Garrido C, Flores S, Pérez C. Diferencias anátomo funcionales y endoscópicas entre la vía aérea del niño y la del adulto. Rev Inst Nal Enf Resp Mex 2007; 20: I42-8.

20. Trippenbach T. Pulmonary reflexes and control of breathing during development. Biol Neonate 1994; 65: 205-10.

21. Rochat I, Leis P, Bouchardy M, Oberli C, Sourial H, Friedli-Burri M, et al. Chest physiotherapy using passive expiratory techniques does not reduce bronchiolitis severity: a randomised controlled trial. Eur J Pediatr 2012; 171: 457-62. 